PROCEEDINGS OF THE

AMERICAN MATHEMATICAL SOCIETY

Volume 124, Number 7, July 1996

\title{
LADDER OPERATORS FOR SZEGÖ POLYNOMIALS AND RELATED BIORTHOGONAL RATIONAL FUNCTIONS
}

\author{
MOURAD E. H. ISMAIL AND MIZAN RAHMAN \\ (Communicated by Palle E. T. Jorgensen)
}

\begin{abstract}
We find the raising and lowering operators for orthogonal polynomials on the unit circle introduced by Szegö and for their four parameter generalization to ${ }_{4} \phi_{3}$ biorthogonal rational functions on the unit circle.
\end{abstract}

\section{INTRODUCTION}

The Szegö theory for orthogonal polynomials on the unit circle [10] was developed in the early part of this century. One aspect of this theory is to relate the limiting behavior of the orthonormal polynomials to the absolutely continuous part of the measure with respect to which the polynomials are orthonormal. As an illustration of his theory Szegö [15] proved that the polynomials $\left\{\mathcal{H}_{n}(z \mid q)\right\}$,

$$
\mathcal{H}_{n}(z \mid q):=\sum_{k=0}^{n} \frac{(q ; q)_{n}}{(q ; q)_{k}(q ; q)_{n-k}}\left(q^{-1 / 2} z\right)^{k},
$$

satisfy the orthogonality relation

$$
\frac{1}{2 \pi i} \int_{|z|=1} \overline{\mathcal{H}_{m}(z \mid q)} \mathcal{H}_{n}(z \mid q) w_{c}(z \mid q) \frac{d z}{z}=q^{-n} \frac{(q ; q)_{n}}{(q ; q)_{\infty}} \delta_{m, n}
$$

where the weight function $w_{c}(z \mid q)$ is given by

$$
w_{c}(z \mid q):=\left(q^{1 / 2} z, q^{1 / 2} / z ; q\right)_{\infty} .
$$

Throughout this paper we shall always assume that $0<q<1$ and shall adopt the following notation for the $q$-shifted factorials [9]

$$
(a ; q)_{0}:=1, \quad(a ; q)_{n}:=\prod_{k=1}^{n}\left(1-a q^{k-1}\right), \quad n=1,2, \ldots, \text { or } \infty
$$

and for the multiple $q$-shifted factorials

$$
\left(a_{1}, a_{2}, \ldots, a_{k} ; q\right)_{n}:=\prod_{j=1}^{k}\left(a_{j} ; q\right)_{n} .
$$

Received by the editors July 5, 1994 and, in revised form, February 2, 1995.

1991 Mathematics Subject Classification. Primary 33D45; Secondary 30E05.

Key words and phrases. Szegö polynomials, $q$-difference operators, orthogonality on the unit circle, $q$-beta integrals, biorthogonal rational functions, raising and lowering operators, $q$-SturmLiouville equations.

Research partially supported by NSF grant DMS 9203659 and NSERC grant A6197. 
We shall also need the notion of basic hypergeometric series

$$
\begin{aligned}
{ }_{r} \phi_{s}\left(\begin{array}{c}
a_{1}, \ldots, a_{r} \\
b_{1}, \ldots, b_{s}
\end{array} \mid q, z\right) & ={ }_{r} \phi_{s}\left(a_{1}, \ldots, a_{r} ; b_{1}, \ldots, b_{s} ; q, z\right) \\
& =\sum_{n=0}^{\infty} \frac{\left(a_{1}, \ldots, a_{r} ; q\right)_{n}}{\left(q, b_{1}, \ldots, b_{s} ; q\right)_{n}} z^{n}\left(-q^{n(n-1) / 2}\right)^{s+1-r} .
\end{aligned}
$$

The subscript $c$ in $w_{c}(z \mid q)$ refers to the unit circle.

There is a hierarchy of the so-called classical $q$-orthogonal polynomials with the Askey-Wilson polynomials ([4], [6], [9]) being the most general and the continuous $q$-Hermite polynomials ([5], [9]) at the bottom of the chart. The polynomials at a certain level are special or limiting cases of some of the polynomials at a higher level. In [7] Berg and Ismail showed how one can use generating functions to go from the continuous $q$-Hermite polynomials to the Askey-Wilson polynomials through the intermediate stage of the Al-Salam-Chihara polynomials [1]. We believe that the Szegö polynomials $\left\{\mathcal{H}_{n}(z \mid q)\right\}$ are the unit circle version of the continuous $q$-Hermite polynomials. The unit circle analogues of the Askey-Wilson polynomials are the four-parameter biorthogonal rational functions introduced by Al-Salam and Ismail in [2]. They are the pair $\left\{r_{n}\right\},\left\{s_{n}\right\}$,

$$
r_{n}(z ; a, \alpha, b, \beta \mid q):={ }_{4} \phi_{3}\left(\begin{array}{c|c}
q^{-n}, a b \alpha \beta q^{n-1}, b q^{1 / 2}, b z \\
b \alpha, b \beta, a b q^{1 / 2} z
\end{array} \mid q, q\right)
$$

and

$$
s_{n}(z ;, a, \alpha, b, \beta \mid q)=r_{n}(z ; \bar{\alpha}, \bar{a}, \bar{\beta}, \bar{b} \mid q) .
$$

Al-Salam and Ismail [2] established the biorthogonality relation

$$
\begin{aligned}
& \frac{1}{2 \pi i} \int_{|z|=1} w_{c}(z ; a, \alpha, b, \beta \mid q) r_{n}(z ; a, \alpha, b, \beta \mid q) \overline{s_{m}(z ; a, \alpha, b, \beta \mid q)} \frac{d z}{z} \\
& \quad=\kappa(a, \alpha, b, \beta) \frac{\left(q, a \alpha, a b \alpha \beta q^{n-1} ; q\right)_{n}}{(b \beta ; q)_{n}(a b \alpha \beta ; q)_{2 n}}(b \beta)^{n} \delta_{m, n},
\end{aligned}
$$

where the weight function $w_{c}(z ; a, \alpha, b, \beta \mid q)$ is

$$
w_{c}(z ; a, \alpha, b, \beta \mid q):=\frac{\left(q^{1 / 2} z, q^{1 / 2} / z, a b q^{1 / 2} z, \alpha \beta q^{1 / 2} / z ; q\right)_{\infty}}{(a z, \alpha / z, b z, \beta / z ; q)_{\infty}}
$$

and the total mass $\kappa(a, \alpha, b, \beta)$ is

$$
\begin{aligned}
\kappa(a, \alpha, b, \beta) & =\frac{1}{2 \pi i} \int_{|z|=1} w_{c}(z ; a, \alpha, b, \beta \mid q) \frac{d z}{z} \\
& =\frac{\left(a q^{1 / 2}, \alpha q^{1 / 2}, b q^{1 / 2}, \beta q^{1 / 2}, a b \alpha \beta ; q\right)_{\infty}}{(q, a \alpha, b \alpha, a \beta, b \beta ; q)_{\infty}} .
\end{aligned}
$$

When $a=\alpha=0$ the functions $r_{n}$ become polynomials previously studied by Pastro [12]. Observe that (1.11) is a special case of (4.11.3) in [9].

The $q$-difference operator $D_{q, z}$ is

$$
\left(D_{q, z} f\right)(z):=\frac{f(z)-f(q z)}{(1-q) z} .
$$

The purpose of this work is first to compute the adjoint of $D_{q, z}$ on a suitable inner product space, then to show that $D_{q, z}$ and its adjoint are, respectively, the lowering 
and raising operators of the Szegö polynomials $\left\{\mathcal{H}_{n}(z \mid q)\right\}$, and finally to generalize these results to the biorthogonal rational functions $\left\{r_{n}\right\}$ and $\left\{s_{n}\right\}$. In Section 2 we shall treat the polynomials $\left\{\mathcal{H}_{n}(z \mid q)\right\}$ and we also find the $q$-Sturm-Liouville system whose eigenfunctions are the Szegö polynomials. This latter fact is then used to give an elementary proof of the orthogonality of the $\mathcal{H}_{n}$ 's with respect to $w_{c}(z \mid q)$. Section 3 develops the $q$-Sturm-Liouville problem associated with $D_{q, z}$ on the unit circle. In Section 4 we identify the raising and lowering operators for the more general and more complicated case of the biorthogonal pair $\left\{r_{n}, s_{n}\right\}$. Section 5 contains a new derivation of the biorthogonality relation (1.9) through the use of raising and lowering operators found in Section 4.

This work is part of the renewed interest in biorthogonal rational functions and polynomials ([3], [11], [13], [14]).

\section{LADDER OPERATORS}

The operator $D_{q, z}$ of $(1.12)$ acts nicely on the $\mathcal{H}_{n}$ 's. It is straightforward to derive

$$
D_{q, z} \mathcal{H}_{n}(z \mid q)=\frac{q^{-1 / 2}\left(1-q^{n}\right)}{1-q} \mathcal{H}_{n-1}(z \mid q)
$$

from the representation (1.1). This shows that $D_{q, z}$ acts as a lowering operator on the $\mathcal{H}_{n}$ 's. In order to find a raising operator we need to compute the adjoint of $D_{q, z}$ with respect to a suitable inner product. Before we introduce the appropriate inner product space we need to introduce some notation. If $f$ is analytic in $\rho_{1}<$ $|z|<\rho_{2}$, then $\bar{f}$ will denote the function whose Laurent coefficients are the complex conjugates of the corresponding Laurent coefficients of $f$. Thus $\bar{f}$ is also analytic in $\rho_{1}<|z|<\rho_{2}$. Consider the set of functions

$$
\mathcal{F}_{\nu}:=\left\{f: f(z) \text { is analytic for } q^{\nu} \leq|z| \leq q^{-\nu}\right\} .
$$

This set will be equipped with the inner product

$$
\langle f, g\rangle_{c}:=\frac{1}{2 \pi i} \int_{|z|=1} f(z) \overline{g(z)} \frac{d z}{z} .
$$

We shall now use $\mathcal{F}_{\nu}$ to denote this inner product space. It is clear that if $f \in \mathcal{F}_{\nu}$, then $\bar{f} \in \mathcal{F}_{\nu}$. Note that $\overline{f(z)}=\bar{f}(\bar{z})$.

Theorem 2.1. The adjoint of the $q$-difference operator $D_{q, z}$ is $T_{q, z}$,

$$
\left(T_{q, z} f\right)(z)=\frac{z[f(z)-q f(q z)]}{1-q}
$$

that is

$$
\left\langle D_{q, z} f, g\right\rangle_{c}=\left\langle f, T_{q, z} g\right\rangle_{c}, \quad \text { for } f, g \in \mathcal{F}_{1}
$$

Proof. We have

$$
\begin{aligned}
\left\langle D_{q, z} f, g\right\rangle_{c} & =\frac{1}{2 \pi i} \int_{|z|=1} \frac{f(z)-f(q z)}{(1-q) z} \overline{g(z)} \frac{d z}{z} \\
& =\frac{1}{2 \pi i} \int_{|z|=1} \frac{f(z)}{(1-q) z} \bar{g}\left(z^{-1}\right) \frac{d z}{z}-\frac{1}{2 \pi i} \int_{|z|=q^{-1}} \frac{f(q z)}{(1-q) z} \bar{g}\left(z^{-1}\right) \frac{d z}{z}
\end{aligned}
$$


since $f$ and $\bar{g}$ are analytic in $1 \leq|z| \leq q^{-1}$. We replace $z$ by $q^{-1} z$ in the last integral. This gives

$$
\begin{aligned}
\left\langle D_{q, z} f, g\right\rangle_{c} & =\frac{1}{2 \pi i} \int_{|z|=1} f(z) \frac{\bar{g}\left(z^{-1}\right)-q \bar{g}\left(q z^{-1}\right)}{(1-q) z} \frac{d z}{z} \\
& =\frac{1}{2 \pi i} \int_{|z|=1} f(z) \frac{\overline{z[g(z)-q g(q z)]}}{(1-q)} \frac{d z}{z}
\end{aligned}
$$

and the proof is complete.

Observe that $T_{q, z}$ can be written in the form

$$
\left(T_{q, z} f\right)(z)=q z^{2}\left(D_{q, z} f\right)(z)+z f(z) .
$$

We now show that $T_{q, z}$ is a raising operator for the polynomials $\mathcal{H}_{n}(z \mid q)$. Since $w_{c}(z \mid q)$ is real on the unit circle, we can rewrite the orthogonality relation (1.2) in the form

$$
\begin{aligned}
q^{-n} \frac{(q ; q)_{n}}{(q ; q)_{\infty}} \delta_{m, n} & =\left\langle\mathcal{H}_{m}(z \mid q), w_{c}(z \mid q) \mathcal{H}_{n}(z \mid q)\right\rangle_{c} \\
& =\frac{q^{1 / 2}(1-q)}{1-q^{m+1}}\left\langle D_{q, z} \mathcal{H}_{m+1}(z \mid q), w_{c}(z \mid q) \mathcal{H}_{n}(z \mid q)\right\rangle_{c} \\
& =\frac{q^{1 / 2}(1-q)}{1-q^{m+1}}\left\langle\mathcal{H}_{m+1}(z \mid q), T_{q, z} w_{c}(z \mid q) \mathcal{H}_{n}(z \mid q)\right\rangle_{c}
\end{aligned}
$$

This shows that

$$
\frac{1}{w_{c}(z \mid q)} T_{q, z} w_{c}(z \mid q) \mathcal{H}_{n}(z \mid q)
$$

is orthogonal to $\mathcal{H}_{n+1}(z \mid q)$ for all $m \neq n$ with respect to the weight function $w_{c}(z \mid q)$. Hence one would expect

$$
\frac{1}{w_{c}(z \mid q)} T_{q, z} w_{c}(z \mid q) \mathcal{H}_{n}(z \mid q)
$$

to be a constant multiple of $\mathcal{H}_{n+1}(z \mid q)$. Thus we have have been led to the following result.

Theorem 2.2. The raising operator for $\left\{\mathcal{H}_{n}\right\}$ is $T_{q, z}$ in the sense

$$
\frac{1}{w_{c}(z \mid q)} T_{q, z}\left(w_{c}(z \mid q) \mathcal{H}_{n}(z \mid q)\right)=\frac{\sqrt{q}}{1-q} \mathcal{H}_{n+1}(z \mid q)
$$

This theorem follows by direct evaluation of the left-hand side of (2.8). The details are straightforward and are omitted.

Theorem 2.3. The Szegö polynomials have the Rodrigues formula

$$
\mathcal{H}_{n}(z \mid q)=\left(q^{-1 / 2}-q^{1 / 2}\right)^{n} \frac{1}{w_{c}(z \mid q)} T_{q, z}^{n}\left(w_{c}(z \mid q)\right) .
$$

Proof. Apply (2.8) repeatedly.

When we combine (2.4) and (2.8) we arrive at the following theorem.

Theorem 2.4. The polynomials $\left\{\mathcal{H}_{n}(z \mid q)\right\}$ satisfy the q-Sturm-Liouville equation

$$
\frac{1}{w_{c}(z \mid q)} T_{q, z}\left(w_{c}(z \mid q) D_{q, z} \mathcal{H}_{n}(z \mid q)\right)=\lambda_{n} \mathcal{H}_{n}(z \mid q)
$$


where

$$
\lambda_{n}=\frac{\left(1-q^{n}\right)}{(1-q)^{2}} .
$$

Observe that the eigenvalues of (2.11) are distinct. Note that the left-hand side of (2.10) is in factored form and as such is an instance of an Infeld-Hull factorization of a second-order $q$-difference operator.

Theorem 2.5. The orthogonality relation (1.2) follows from (2.1) and (2.8).

Proof. Set

$$
\zeta_{n}=\left\langle w_{c}(z \mid q) \mathcal{H}_{n}(z \mid q), \mathcal{H}_{n}(z \mid q)\right\rangle_{c}
$$

Clearly we have

$$
\begin{aligned}
\frac{q^{-1 / 2}\left(1-q^{n+1}\right)}{1-q} \zeta_{n} & =\left\langle D_{q, z} \mathcal{H}_{n+1}(z \mid q), w_{c}(z \mid q) \mathcal{H}_{n}(z \mid q)\right\rangle_{c} \\
& =\left\langle\mathcal{H}_{n+1}(z \mid q), T_{q, z} w_{c}(z \mid q) \mathcal{H}_{n}(z \mid q)\right\rangle_{c} \\
& =\frac{q^{1 / 2}}{1-q} \zeta_{n+1} .
\end{aligned}
$$

Therefore $\zeta_{n+1}=q^{-1}\left(1-q^{n+1}\right) \zeta_{n}$ and we get

$$
\zeta_{n}=q^{-n}(q ; q)_{n} \zeta_{0} .
$$

To find $\zeta_{0}$ expand $w_{c}(z \mid q)$ using the Jacobi triple product identity [9, (II.28)]

$$
\sum_{-\infty}^{\infty} q^{n^{2}} z^{n}=\left(q^{2},-q z,-q / z ; q^{2}\right)_{\infty},
$$

then substitute the expansion in

$$
\frac{1}{2 \pi i} \int_{|z|=1} w_{c}(z \mid q) \frac{d z}{z} .
$$

It is then straightforward to see that $\zeta_{0}=1 /(q ; q)_{\infty}$. Now substitute for $\zeta_{0}$ in $(2.13)$ to obtain (1.2), and this completes the proof.

\section{3. $q$-STURM LiOUVILLE OPERATORS}

Motivated by equation (2.9) we now consider the more general operator

$$
(M f)(z):=\frac{1}{\omega(z)}\left(T_{q, z}\left(p(z) D_{q, z} f\right)\right)(z),
$$

where $\omega(z)$ is real on the unit circle with some restrictions on $p$ and $\omega$ to follow. The analysis in this section follows closely the plan in [8], where the spectral theory of the Askey-Wilson polynomials is discussed.

Let $\mathcal{H}_{\omega}$ denote the inner product space $L^{2}$ of the unit circle equipped with the inner product

$$
(f, g)_{\omega}:=\frac{1}{2 \pi i} \int_{|z|=1} f(z) \overline{g(z)} \omega(z) \frac{d z}{z},
$$

and let

$$
T:=M_{\mid \mathcal{F}_{2}} \text { in } \mathcal{H}_{w} .
$$


We shall assume that $p$ and $\omega$ satisfy

(i) $\quad p(z)>0$ a.e. on $|z|=1, p \in \mathcal{F}_{1}, 1 / p \in L(\{z:|z|=1\})$;

(ii) on the unit circle $\omega(z)>0$ a.e. and both $\omega$ and $1 / \omega$ are integrable.

The expression $M f$ is therefore defined for $f \in \mathcal{F}_{2}$, and the operator $T$ acts in $\mathcal{H}_{w}$. Furthermore, the domain $\mathcal{F}_{2}$ of $T$ is dense in $\mathcal{H}_{\omega}$ since it contains all polynomials and Laurent polynomials.

Theorem 3.1. The operator $T$ is symmetric in $\mathcal{H}_{\omega}$ and $T \geq 0$.

Proof. For all $f, g \in \mathcal{F}_{2}$, it follows that $(T f, g)_{w}=(f, T g)_{w}$, hence the symmetry of $T$. If $f \in \mathcal{F}_{2}$, then

$$
\begin{aligned}
(f, T f)_{\omega} & =\left\langle f, T_{q, z}\left(p(z) D_{q, z} f\right)\right\rangle_{c}=\left\langle D_{q, z} f, p(z) D_{q, z} f\right\rangle \\
& =\frac{1}{2 \pi i} \int_{|z|=1} p(z)\left|D_{q, z} f(z)\right|^{2} \frac{d z}{z}
\end{aligned}
$$

which proves that $T \geq 0$ and completes the proof of our theorem.

Corollary 3.2. Let $y_{1}, y_{2} \in \mathcal{F}_{2}$ be solutions to

$$
\frac{1}{\omega(z)}\left(T_{q, z}\left(p(z) D_{q, z} f\right)\right)(z)=\lambda f,
$$

with $\lambda=\lambda_{1}$ and $\lambda=\lambda_{2}$, respectively, and assume $\lambda_{1} \neq \lambda_{2}$. Then $y_{1}$ and $y_{2}$ are orthogonal in the sense

$$
\frac{1}{2 \pi i} \int_{|z|=1} y_{1}(z) y_{2}(z) \frac{d z}{z}=0 .
$$

Furthermore, the eigenvalues of (3.5) are all real.

Note that Theorem 3.1 implies that the eigenvalues of $T$ are nonnegative.

Let $\mathcal{Q}(T)$ denote the form domain of $T$ and let $\tilde{T}$ be its Friedrichs extension. Recall that the domain of $T, \mathcal{Q}(T)$, is the completion of $\mathcal{F}_{1}$ with respect to $\|\cdot\|_{\mathcal{Q}}$, where

$$
\|f\|_{\mathcal{Q}}^{2}:=\frac{1}{2 \pi i} \int_{|z|=1} p(z)\left|D_{q, z} f\right|^{2} \frac{d z}{z}+\|f\|_{\omega}^{2},
$$

and if $(., .)_{\mathcal{Q}}$ denotes the inner product on $\mathcal{Q}(T)$, then for all $f \in \mathcal{D}(\tilde{T})$ and $g \in$ $\mathcal{Q}(T)$,

$$
(f, g)_{\mathcal{Q}}=(\tilde{T} f, g)_{\omega} .
$$

We have that $f \in \mathcal{Q}(T)$ if and only if there exists a sequence $\left\{f_{n}\right\} \subset \mathcal{F}_{1}$ such that $\left\|f-f_{n}\right\|_{\mathcal{Q}} \rightarrow 0$; hence $\left\|f-f_{n}\right\|_{w} \rightarrow 0$ and $\left\{\mathcal{D}_{q} f_{n}\right\}$ is a Cauchy sequence in $L^{2}\left(\{z:|z|=1\} ; p(z) \frac{d z}{2 \pi i z}\right)$, with limit $F$ say. From (3.3) and (2.3) it follows that for $\phi \in \mathcal{F}_{1}$,

$$
\begin{aligned}
\frac{1}{2 \pi i} \int_{|z|=1} F(z) \phi(z) \frac{d z}{z} & =\lim _{n \rightarrow \infty} \frac{1}{2 \pi i} \int_{|z|=1}\left(D_{q, z} f_{n}\right)(z) \phi(z) \frac{d z}{z} \\
& =\lim _{n \rightarrow \infty} \frac{1}{2 \pi i} \int_{|z|=1} f_{n}(z) D_{q, z} \phi(z) \frac{d z}{z} \\
& =\frac{1}{2 \pi i} \int_{|z|=1} f(z) D_{q, z} \phi(z) \frac{d z}{z}
\end{aligned}
$$


Thus in analogy with distributional derivatives, we shall say that $F=D_{q, z} f$ in the generalized sense. We conclude that the norm on $\mathcal{Q}(T)$ is defined by (3.7) with $D_{q, z} f$ now defined in the generalized sense. Also, it follows in a standard way that

$$
\begin{aligned}
& \mathcal{D}\left(T^{*}\right)=\left\{f: f, M f \in \mathcal{H}_{\omega}\right\}, \quad T f=M f, \\
\mathcal{D}(\tilde{T})= & \mathcal{Q}(T) \cap \mathcal{D}\left(T^{*}\right) \\
= & \left\{f: p^{1 / 2} D_{q, z} f \in L^{2}(\{z:|z|=1\}), M f \in \mathcal{H}_{\omega}\right\} .
\end{aligned}
$$

\section{Biorthogonal FunCtions}

We first evaluate the action of $D_{q, z}$ on $r_{n}(z ; a, \alpha, b, \beta \mid q)$. A calculation gives

$$
D_{q, z} \frac{(b z ; q)_{k}}{\left(a b q^{1 / 2} z ; q\right)_{k}}=-\frac{b\left(1-a q^{1 / 2}\right)\left(1-q^{k}\right)}{1-q} \frac{(q b z ; q)_{k-1}}{\left(a b q^{1 / 2} z ; q\right)_{k+1}} .
$$

Therefore

$$
\begin{aligned}
\left(a b q^{1 / 2} z ; q\right)_{2} D_{q, z} r_{n}(z ; a, \alpha, b, \beta \mid q)= & \frac{b q^{1-n}\left(1-a q^{1 / 2}\right)\left(1-b q^{1 / 2}\right)\left(1-q^{n}\right)}{(1-q)(1-b \alpha)(1-b \beta)} \\
& \times\left(1-a b \alpha \beta q^{n-1}\right) r_{n-1}(z ; q a, \alpha, b q, \beta \mid q) .
\end{aligned}
$$

This shows that the operator $\left(a b q^{1 / 2} z ; q\right)_{2} D_{q, z}$ is a lowering operator for the $r_{n}$ 's. To guess at the raising operator we use (2.5). Clearly

$$
\begin{aligned}
& \left\langle\left(a b q^{1 / 2} z ; q\right)_{2} D_{q, z} f, w_{c}(1 / z ; q \bar{a}, \bar{\alpha}, q \bar{b}, \bar{\beta} \mid q) g\right\rangle_{c} \\
& \quad=\left\langle D_{q, z} f,\left(\bar{a} \bar{b} q^{1 / 2} / z ; q\right)_{2} w_{c}(1 / z ; q \bar{a}, \bar{\alpha}, q \bar{b}, \bar{\beta} \mid q) g\right\rangle_{c} \\
& \left.\quad=\left\langle f, T_{q, z}\left(\left(\bar{a} \bar{b} q^{1 / 2} / z ; q\right)_{2} w_{c}(1 / z ; q \bar{a}, \bar{\alpha}, q \bar{b}, \bar{\beta} \mid q)\right) g\right)\right\rangle_{c} .
\end{aligned}
$$

This relationship, (4.1) and the orthogonality relation (1.9) show that when $m \neq n$ then

$$
\begin{aligned}
0= & \left\langle r_{m-1}(z ; q a, \alpha, q b, \beta \mid q) w_{c}(z ; q a, \alpha, q b, \beta \mid q), s_{n-1}(z ; q a, \alpha, q b, \beta \mid q)\right\rangle_{c} \\
= & \left\langle r_{n}(z ; a, \alpha, b, \beta \mid q),\right. \\
& \left.T_{q, z}\left(\left(\bar{a} \bar{b} q^{1 / 2} / z ; q\right)_{2} w_{c}(1 / z ; q \bar{a}, \bar{\alpha}, q \bar{b}, \bar{\beta} \mid q) s_{n-1}(z ; q a, \alpha, q b, \beta \mid q)\right)\right\rangle_{c} .
\end{aligned}
$$

But (1.10) shows the symmetry of the weight function

$$
w_{c}(1 / z ; a, \alpha, b, \beta \mid q)=w_{c}(z ; a, \alpha, b, \beta \mid q) .
$$

Now (4.2), (4.3), (1.8) and the biorthogonality relation (1.9) suggest that

$$
T_{q, z}\left(\left(\bar{a} \bar{b} q^{1 / 2} / z ; q\right)_{2} w_{c}(z ; q \bar{a}, \bar{\alpha}, q \bar{b}, \bar{\beta} \mid q) s_{n-1}(z ; q a, \alpha, q b, \beta \mid q)\right)
$$

is a multiple of

$$
w_{c}(1 / z ; \bar{a}, \bar{\alpha}, \bar{b}, \bar{\beta} \mid q) s_{n}(z ; q a, \alpha, q b, \beta \mid q) .
$$

This suggests that the raising operator we are looking for is defined by

$$
\left(L^{+} f\right)(z)=\frac{1}{w_{c}(z ; a, \alpha, b, \beta \mid q)} T_{q, z}\left(\left(\alpha \beta q^{-3 / 2} / z ; q\right)_{2} w_{c}(z ; a, \alpha, b, \beta \mid q) f(z)\right) .
$$


Observe that $L^{+}$of (4.5) is a raising operator when it acts on $r_{n}$ because the functions $\left\{r_{n}, s_{n}\right\}$ are biorthogonal and not necessarily orthogonal. Like the case of the Szegö polynomials it turns out that we have made the correct guess.

Theorem 4.1. The raising and lowering operators for $\left\{r_{n}(z ; a, \alpha, b, \beta \mid q)\right\}$ are $L^{+}$ and $L^{-}$, where

$$
\left(L^{-} f\right)(z):=\left(\left(\alpha \beta q^{1 / 2} z ; q\right)_{2} D_{q, z} f\right)(z),
$$

and $L^{+}$is as in (4.5). In other words

$$
\begin{aligned}
L^{-} r_{n}(z ; a, \alpha, b, \beta \mid q)= & \frac{b q^{1-n}\left(1-q^{1 / 2} a\right)\left(1-q^{1 / 2} b\right)\left(1-a b \alpha \beta q^{n-1}\right)}{(1-q)(1-b \alpha)(1-b \beta)} \\
& \times\left(1-q^{n}\right) r_{n-1}(z ; q a, \alpha, q b, \beta \mid q)
\end{aligned}
$$

and

$$
L^{+} r_{n-1}(z ; a, \alpha, b, \beta \mid q)=\frac{(1-b \alpha / q)(1-b \beta / q)}{(1-q) b} r_{n}(z ; a, \alpha / q, b, \beta / q \mid q)
$$

Proof. We need only to prove (4.8). Set

$$
\begin{aligned}
& \left.T_{q, z}\left(\left(\alpha \beta q^{1 / 2} / z ; q\right)\right)_{2} w_{c}(z ; a, q \alpha, b, q \beta \mid q) r_{n-1}(z ; a, q \alpha, b, q \beta \mid q)\right) \\
& \quad=\frac{z}{1-q} w_{c}(z ; a, \alpha, b, \beta \mid q) L_{n}(z) .
\end{aligned}
$$

A calculation using (4.9), (1.7) and (1.10) gives

$$
\begin{aligned}
& L_{n}(z)=(1-\alpha / z)(1-\beta / z)_{4} \phi_{3}\left(\begin{array}{c}
q^{1-n}, a b \alpha \beta q^{n}, q^{1 / 2} b, b z \\
q b \alpha, q b \beta, a b q^{1 / 2} z
\end{array} \mid q, q\right) \\
& \quad+\frac{\left(z q^{1 / 2}-\alpha \beta\right)(1-a z)(1-b z)}{z^{2}\left(1-a b q^{1 / 2} z\right)}{ }_{4} \phi_{3}\left(\begin{array}{c}
q^{1-n}, a b \alpha \beta q^{n}, q^{1 / 2} b, q b z \\
a b q^{3 / 2} z, q b \alpha, q b \beta
\end{array} \mid q, q\right) .
\end{aligned}
$$

Recall the Sears transformation [9, (III.15)]

$$
\begin{aligned}
{ }_{4} \phi_{3} & \left(\begin{array}{c}
q^{-n}, A, B, C \\
D, E, F
\end{array} \mid q, q\right) \\
& =\frac{(E / A, F / A ; q)_{n}}{(E, F ; q)_{n}} A^{n}{ }_{4} \phi_{3}\left(\begin{array}{c}
q^{-n}, A, D / B, D / C \\
D, A q^{1-n} / E, A q^{1-n} / F
\end{array}\right.
\end{aligned}
$$

where $A B C q^{1-n}=D E F$. Now apply (4.11) with the invariant parameters $A$ and $D$ being the terms depending on $z$. After some routine calculations we obtain

$$
\begin{aligned}
& \frac{(q b \alpha, q b \beta ; q)_{n-1}}{\left(q^{1-n} z / \alpha, q^{1-n} z / \beta ; q\right)_{n}}\left(\frac{z}{\alpha \beta b q^{n-1}}\right)^{n-1} \frac{z^{2}}{\alpha \beta} L_{n}(z) \\
& =q^{n-1} \sum_{k=0}^{n-1} \frac{\left(q^{1-n}, a z, b z, q^{-n+1 / 2} z / \alpha \beta ; q\right)_{k}}{\left(q, q^{1-n} z / \alpha, q^{1-n} z / \beta, a b q^{1 / 2} z ; q\right)_{k}} q^{k} \\
& \quad-\frac{\left(\alpha \beta-q^{1 / 2} z\right)}{\left(\alpha \beta-q^{-n+1 / 2} z\right)} \sum_{k=0}^{n-1} \frac{\left(q^{1-n} ; q\right)_{k}\left(a z, b z, q^{-n+1 / 2} z / \alpha \beta ; q\right)_{k+1}}{(q ; q)_{k}\left(q^{1-n} z / \alpha, q^{1-n} z / \beta, a b q^{1 / 2} z ; q\right)_{k+1}} q^{k}
\end{aligned}
$$


In the first sum in the above equation isolate the $k=0$ term from the remaining terms, but in the second sum isolate the $k=n-1$ term from the rest of the series; then combine the two remaining series. A fantastic cancellation occurs and we obtain

$$
\begin{array}{r}
T_{q, z}\left[\left(\alpha \beta q^{1 / 2} / z ; q\right)_{2} w_{c}(z ; a, q \alpha, b, q \beta \mid q) r_{n-1}(z ; a, q \alpha, b, q \beta \mid q)\right] \\
=\frac{(1-b \alpha)(1-b \beta / q)}{(1-q) b} w_{c}(z ; a, \alpha, b, \beta \mid q) r_{n}(z ; a, \alpha, b, \beta \mid q),
\end{array}
$$

which is equivalent to (4.8) and the proof is complete.

\section{The BiorthogonAlity RELATiOn}

Let us denote

$$
\begin{aligned}
& I_{m, n}(a, \alpha, b, \beta) \\
& \quad:=\frac{1}{2 \pi i} \int_{|z|=1} w_{c}(z ; a, \alpha, b, \beta \mid q) r_{n}(z ; a, \alpha, b, \beta \mid q) \overline{s_{m}(z ; a, \alpha, b, \beta \mid q)} \frac{d z}{z},
\end{aligned}
$$

which is just the integral on the left-hand side of (1.9). Observing that

$$
\left\langle T_{q, z} f(z), g(z)\right\rangle_{c}=\left\langle f(z), D_{q, z} g(z)\right\rangle_{c}
$$

we use (4.12) to write

$$
\begin{aligned}
& \frac{(1-\alpha b)(1-\beta b)}{b(1-q)} I_{m, n}(a, \alpha, b, \beta) \\
& =\frac{1}{2 \pi i} \int_{|z|=1}\left(\alpha \beta q^{1 / 2} / z ; q\right)_{2} w_{c}(z ; a, q \alpha, b, q \beta \mid q) r_{n-1}(z ; a, q \alpha, b, q \beta \mid q) \\
& \quad \times \frac{D_{q, z} s_{m}(z ; a, \alpha, b, \beta \mid q)}{\frac{d z}{z}} \\
& =\beta q^{1-m} \frac{\left(1-\alpha q^{1 / 2}\right)\left(1-\beta q^{1 / 2}\right)\left(1-q^{m}\right)\left(1-a b \alpha \beta q^{m-1}\right)}{(1-q)(1-a \beta)(1-b \beta)} \\
& \quad \times \frac{1}{2 \pi i} \int_{|z|=1} w_{c}(z ; a, q \alpha, b, q \beta \mid q) r_{n-1}(z ; a, q \alpha, b, q \beta \mid q) \overline{s_{m-1}(z ; a, q \alpha, b, q \beta \mid q)} \frac{d z}{z}
\end{aligned}
$$

by $(1.7),(1.8)$ and $(4.1)$. Hence

$$
\begin{aligned}
I_{m, n}(a, \alpha, b, \beta)= & -b \beta q \frac{\left(1-q^{1 / 2} \alpha\right)\left(1-q^{1 / 2} \beta\right)\left(1-q^{-m}\right)\left(1-a \alpha b \beta q^{m-1}\right)}{(1-\alpha b)(1-a \beta)(1-b \beta)^{2}} \\
& \times I_{m-1, n-1}(a, q \alpha, b, q \beta) .
\end{aligned}
$$

Suppose $m \geq n$. Then iterating (5.3) $n-1$ times we obtain

$$
\begin{aligned}
I_{m, n}(a, \alpha, b, \beta)=( & -b \beta)^{n} q^{\left({ }_{2}^{n+1}\right)} \frac{\left(q^{1 / 2} \alpha, q^{1 / 2} \beta, q^{-m}, a \alpha b \beta q^{m-1} ; q\right)_{n}}{(\alpha b, a \beta, b \beta, b \beta ; q)_{n}} \\
& \times I_{m-n, 0}\left(a, q^{n} \alpha, b, q^{n} \beta\right)
\end{aligned}
$$


If $m>n$, then using the identity

$$
\begin{aligned}
T_{q, \bar{z}}\left[\left(a b q^{1 / 2} z ; q\right)_{2} w(\bar{z} ; \alpha, q a, \beta, q b \mid q) \overline{s_{k-1}(z ; q a, \alpha, q b, \beta \mid q)}\right] \\
\quad=\frac{(1-a \beta)(1-b \beta)}{a b \beta(1-q)} w(z ; a, \alpha, b, \beta \mid q) \overline{s_{k}(z ; a, \alpha, b, \beta \mid q)}
\end{aligned}
$$

gives

$$
\begin{aligned}
& w\left(z ; a, q^{n} \alpha, b, q^{n} \beta \mid q\right) \overline{s_{m-n}\left(z ; a, q^{n} \alpha, b, q^{n} \beta \mid q\right)} \\
& \quad=\frac{\left(a b \beta q^{n}(1-q)\right.}{\left(1-a \beta q^{n}\right)\left(1-b \beta q^{n}\right)} \\
& \quad \times T_{q, \bar{z}}\left[\left(q^{1 / 2} a b z ; q\right)_{2} w\left(\bar{z} ; q^{n} \alpha, q a, q^{n} \beta, q b \mid q\right) \overline{s_{m-n}\left(z ; q a, q^{n} \alpha, q b, q^{n} \beta \mid q\right)}\right] .
\end{aligned}
$$

But the integral over the expression on the right-hand side of (5.6) over the unit circle vanishes. This proves the orthogonality when $m>n$. If $m=n$, then we have

$$
\begin{aligned}
I_{0,0}\left(a, q^{n} \alpha, b, q^{n} \beta\right) & =\frac{1}{2 \pi i} \int_{|z|=1} w\left(z ; a, q^{n} \alpha, b, q^{n} \beta \mid q \frac{d z}{z}\right. \\
& =\kappa(a, \alpha, b, \alpha) \frac{(a \alpha, b \alpha, a \beta, b \beta ; q)_{n}}{\left(q^{1 / 2} \alpha, q^{1 / 2} \beta ; q\right)_{n}(a b \alpha \beta ; q)_{2 n}},
\end{aligned}
$$

by (1.11). On the other hand, if $m<n$, then iterating (5.3) $m-1$ times we obtain

$$
\begin{aligned}
I_{m, n}(a, \alpha, b, \beta)= & (-b \beta)^{m} \frac{\left(q^{1 / 2} \alpha, q^{1 / 2} \beta, q^{-m}, a b \alpha \beta q^{m-1} ; q\right)_{m}}{(\alpha b, a \beta, b \beta, b \beta ; q)_{m}} q^{\left(\begin{array}{c}
m+1 \\
2
\end{array}\right)} \\
& \times I_{0, n-m}\left(a, q^{m} \alpha, b, q^{m} \beta\right) \\
= & (-b \beta)^{m} \frac{\left(q^{1 / 2} \alpha, q^{1 / 2} \beta, q^{-m}, a b \alpha \beta q^{m-1} ; q\right)_{m}}{(\alpha b, a \beta, b \beta, b \beta ; q)_{m}} q^{\left(\begin{array}{c}
m+1 \\
2
\end{array}\right)} \\
& \times \frac{1}{2 \pi i} \int_{|z|=1} w\left(z ; a, q^{m} \alpha, b, q^{m} \beta \mid q\right) r_{n-m}\left(z ; a, q^{m} \alpha, b, q^{m} \beta \mid q\right) \frac{d z}{z} .
\end{aligned}
$$

However, by (4.12),

$$
\begin{aligned}
& w\left(z ; a, q^{m} \alpha, b, q^{m} \beta \mid q\right) r_{n-m}\left(z ; a, q^{m} \alpha, b, q^{m} \beta \mid q\right) \\
& =\frac{b(1-q)}{\left(1-q^{m} \alpha b\right)\left(1-q^{m} b \beta\right)} \\
& \quad \times T_{q, z}\left[\left(\alpha \beta q^{2 m+1 / 2} / z ; q\right)_{2} w\left(z ; a, q^{m+1} \alpha, b, q^{m+1} \beta \mid q\right)\right. \\
& \left.\quad \times r_{n-m-1}\left(z ; a, q^{m+1} \alpha, b, q^{m+1} \beta \mid q\right)\right]
\end{aligned}
$$

and the integral of the right-hand side over the unit circle is zero. So, $I_{m, n}(a, \alpha, b, \beta)$ $=0$ when $m<n$. This together with (5.6) and (5.7) constitute an alternate proof of the biorthogonality relation (1.9), which is based on the use of the raising and lowering operators $L^{ \pm}$. 


\section{REFERENCES}

1. W. A. Al-Salam and T. S. Chihara, Convolutions of orthogonal polynomials, SIAM J. Math. Anal. 7 (1976), 16-28. MR 53:3381

2. W. A. Al-Salam and M. E. H. Ismail, A q-beta integral on the unit circle and some biorthogonal rational functions, Proc. Amer. Math. Soc. 121 (1994), 553-561. MR 94h:33011

3. W. A. Al-Salam and A. Verma, Q-analogs of some biorthogonal functions, Canad. Math. Bull. 26(1983), 225-227. MR 84e:33010

4. G. E. Andrews and R. A. Askey, Classical orthogonal polynomials, in "Polynomes Orthogonaux et Applications", eds. C. Breziniski et ál., Lecture Notes in Mathematics, vol. 1171, Springer-Verlag, Berlin, 1984, pp. 36-63.

5. R. A. Askey and M. E. H. Ismail, A generalization of ultraspherical polynomials, in "Studies in Pure Mathematics", ed. P. Erdös, Birkhauser, Basel, 1983, pp. 55-78. MR 87a:33015

6. R. A. Askey and J. A. Wilson, Some basic hypergeometric orthogonal polynomials that generalize Jacobi polynomials, Memoirs Amer. Math. Soc. Number 319 (1985). MR 87a:05023

7. C. Berg and M. E. H. Ismail, Q-Hermite polynomials and classical orthogonal polynomials, Canad. J. Math. (1996), to appear.

8. W. D. Evans, B. M. Brown and M. E. H. Ismail, The Askey-Wilson polynomials and q-SturmLiouville problems, Math. Proc. Camb. Philos. Soc. 119 (1996), 1-16.

9. G. Gasper and M. Rahman, Basic Hypergeometric Series, Cambridge University Press, Cambridge, 1990. MR 91d:33034

10. U. Grenander and G. Szegö, Toeplitz Forms and Their Applications, University of California Press, Berkely, 1958, reprinted by Chelsea, Bronx, 1984. MR 88b:42031

11. M. E. H. Ismail and D. R. Masson, Q-Hermite polynomials, biorthogonal rational functions, Transactions Amer. Math. Soc. 346 (1994), 63-110. CMP 94:16

12. P. I. Pastro, Orthogonal polynomials and some q-beta integrals of Ramanujan, J. Math. Anal. Appl. 112 (1985), 517-540. MR 87c:33015

13. M. Rahman, Biorthogonality of a system of rational functions with respect to a positive measure on $[-1,1]$, SIAM J. Math. Anal. 22 (1991), 1421-1431. MR 92h:33016

14. M. Rahman and S. K. Suslov, Classical biorthogonal rational functions in "Methods of Approximation Theory in Complex Analysis and Mathematical Physics", A. A. Goncar and E. B. Saff, editors, Lecture Notes in Mathematics 1550, Springer-Verlag, Berlin, pp. 131-150.

15. G. Szegő, Beitrag zur Theorie der Thetafunktionen, Sitz. Preuss. Akad. Wiss. Phys. Math. Kl., XIX (1926), 242-252, Reprinted in "Collected Papers", edited by R. Askey, Volume I, Birkhauser, Boston, 1982.

Department of Mathematics, University of South Florida, Tampa, Florida 33620

Department of Mathematics, Carleton University, Ottawa, Ontario, Canada K1S 5B6 